\title{
Application of Cervical Vestibular-Evoked Myogenic Potentials in Adults with Moderate to Profound Sensorineural Hearing Loss: A Preliminary Study
}

\author{
Francesco Ciodaro ${ }^{1}$ Francesco Freni ${ }^{1}$ Giuseppe Alberti ${ }^{1}$ Marco Forelli ${ }^{1}$ Francesco Gazia ${ }^{1}$ \\ Rocco Bruno ${ }^{1}$ Enrique Perello Sherdell ${ }^{2}$ Bruno Galletti ${ }^{1}$ Francesco Galletti ${ }^{1}$
}

\footnotetext{
${ }^{1}$ Department of Human Pathology in Adult and Developmental Age "Gaetano Barresi," Unit of Otorhinolaryngology, University of Messina, Messina ME, Italy

2 Otorhinolaryngology Service, Hospital Universitario Vall d'Hebron, Barcelona, Spain
}

\begin{abstract}
Address for correspondence Francesco Gazia, MD, Department of Human Pathology in Adult and Developmental Age "Gaetano Barresi," Unit of Otorhinolaryngology, University of Messina, Via Consolare Valeria 1, Messina ME 98125, Italy (e-mail: ssgazia@gmail.com).
\end{abstract}

Int Arch Otorhinolaryngol 2020;24:e5-e10.

\begin{abstract}
Keywords

- hearing loss

- bacterial meningitis

- vestibular-evoked myogenic potentials

Introduction The cochlea and the vestibular receptors are closely related in terms of anatomy and phylogeny. Patients with moderate to profound sensorineural hearing loss (MPSHL) should have their vestibular organ functions tested.

Objective To evaluate the incidence of vestibular abnormalities in patients with MPSHL and to study the correlation between the etiology of hearing loss ( $\mathrm{HL}$ ) and a possible damage to the labyrinth.

Methods A case-control retrospective study was performed. In the case group, 20 adults with MPSHL of known etiology were included. The control group was composed of 15 adults with normal hearing. The case group was divided into 4 subgroups based on the etiology (bacterial meningitis, virus, vascular disease, congenital). Cervical vestibular-evoked myogenic potentials (cVEMPs) were used to rate the saccular function and lower vestibular nerve.

Results The study was performed in 70 ears, and it highlighted the presence of early biphasic P1-N1 complex in 29 (71.5\%) out of 40 ears in the study group, and in all of the 30 ears in the control group $(p=0.001)$. Regarding the presence or absence of cVEMPs among the four subgroups of patients with MPSHL, the data were statistically significant $(p<0.001)$. The comparison between the latencies and amplitude of P1$\mathrm{N} 1$ in case and control groups from other studies and in the four subgroups of cases in the present study did not detect statistically significant differences.

Conclusion The present study demonstrates that patients with MPSHL have a high incidence of damage to the labyrinthine organs, and it increases the current knowledge about the etiopathogenesis of sensorineural $\mathrm{HL}$, which is often of unknown nature.
\end{abstract}

\section{Introduction}

The cochlea, the vestibular receptors, the semi-circular canals, and the otolith organs are closely related in terms of anatomy and phylogeny. They share the continuous mem- branous labyrinth of the inner ear, have similar receptor cells, and are supplied by a common arterial vessel, the labyrinthine artery, which arises from the anterior inferior cerebellar artery (AICA). ${ }^{1}$ It is reasonable to hypothesize that inner ear diseases may affect both the vestibular system and received

February 3, 2019

accepted

July 27, 2019
DOI https://doi.org/

10.1055/s-0039-1697988.

ISSN $1809-9777$.
Copyright (c) 2020 by Thieme Revinter

Publicações Ltda, Rio de Janeiro, Brazil
License terms

() (1) $\odot \circledast$ 
the cochlea, or, in other words, that people with cochlear hearing damage may also have vestibular deficiency. Therefore, patients with moderate to profound sensorineural hearing loss (MPSHL) should have their vestibular organ functions tested. ${ }^{2} \mathrm{~A}$ comprehensive evaluation of the extent of the vestibular lesions involved in MPSHL may be useful to understand the range and extent of inner ear lesions, and provide some tips on the potential pathogenesis. ${ }^{3}$ In recent years, there has been a growing awareness of vestibular dysfunction in people with hearing loss (HL). The cervical vestibular-evoked myogenic potentials (cVEMPs) is an objective and non-invasive test, which allows the rating of saccular function and lower vestibular nerve. Particular sounds, sent to the ears at a certain frequency and intensity, stimulate a reflex contraction and subsequent release of the neck muscles, specifically the sternocleidomastoid muscle (SCM), in response to the excitation of the saccule; this is called vestibulo-collic reflex. The cVEMP response is recorded as a bioelectric potential variation, with the appearance of two wave patterns. ${ }^{1}$

There are different causes of MPSHL that are encountered in the otology clinical practice. Congenital HL can be syndromic or non-syndromic. The major risk factors for congenital HL include consanguinity (genetic causes) or intrauterine infections, such as maternal rubella or cytomegalovirus (CMV), that cause bilateral MPSHL in children. ${ }^{4}$ The etiological factors of acquired MPSHL are varied. A previously reported analysis of 310 adult cases included meningitis, viruses, vascular diseases, idiopathic sudden sensorineural $\mathrm{HL}$, chronic suppurative otitis media, trauma, ototoxic medications, and unknown etiology as causes of acquired MPSHL. ${ }^{5-11}$

We proposed the present study to increase the current knowledge about the etiopathogenesis of sensorineural HL, which is often of unknown nature.

The aim of the present study was to evaluate the incidence of vestibular abnormalities in patients with MPSHL. Another purpose was to study the correlation between the etiology of $\mathrm{HL}$ and a possible alteration in labyrinthine function.

\section{Materials and Methods}

We performed a case-control retrospective study. In the case group, 20 people with the following inclusion criteria were enrolled: patients older than 18 years with MPSHL of known etiology, and type-A tympanograms. The exclusion criteria were: syndromic patients, deafness caused by stapedial or cochlear otosclerosis, and patients with previous ear surgery. The control group was composed of 15 people older than 18 years of age, with normal hearing and type-A tympanograms.

The case group was divided into four subgroups based on the etiology ( $\mathbf{-}$ Table $\mathbf{1}$ ). They were composed of:

- two patients with HL after bacterial meningitis, diagnosed by lumbar puncture; ${ }^{12}$

- four patients with HL after viral infection in childhood; ${ }^{13}$

- six patients with congenital non-syndromic $\mathrm{HL} ;{ }^{14}$ and
- eight patients with HL and previous ischemic episodes. These patients had cardiovascular and thrombophilic risk factors (hypercholesterolemia, hyperhomocysteinemia, high factor VIII, elevated plasminogen activator inhibitor-1 levels, and anticardiolipin antibodies). ${ }^{15,16}$

Pure tone audiometry was performed to determine the minimum audibility with a range of 125 to $8,000 \mathrm{~Hz}$ by air, and with a range of 250 to $4,000 \mathrm{~Hz}$ for bone conduction. The pure tone average (PTA) of the hearing threshold levels at a set of specified frequencies $(500,1,000,2,000$, and $4,000 \mathrm{~Hz})$ was computed. The 2010 version of the Neuro-Audio amplifier (Neurosoft, Ivanovo, Ivanovo Oblast, Russia) was used to measure the CVEMPs.

The cVEMPs were measured with the subject sitting down. The electrodes were well positioned after adequately cleaning the skin. The results were negative in the middle third of the SCM; positive in the middle of the manubrium; and common in the forehead.

To activate the SCM, during the exam, it is necessary that the subject tone up muscle turning his/her head to the side opposite to the one being tested. The parameters of the stimuli used are tone burst to $500 \mathrm{~Hz}$ with 4 -ms rise and fall times and 0 -ms plateau, with a stimulation rate of $5.1 / \mathrm{s}$, in rarefaction polarity. The acquisition parameters are filtering $5 / 500 \mathrm{~Hz}$ in a 50 -ms window of time, with an impedance lower than $5 \mathrm{k} \Omega$. The examination was performed by sending to the ears a stimulus of $105 \mathrm{~dB}$ NHL. We calculated the presence of the early biphasic P1-N1 complex and its latency (ms) and amplitude $(\mu \mathrm{v})$. The cVEMP response consists of an initial positivity (P1) followed by a negativity (N1). It is an evoked potential. Although $\mathrm{P} 1$ is positive, it is deemed negative on many cVEMPs because of electrode placement (basically putting them on backwards). The most reliable measure of the cVEMP response is the amplitude. ${ }^{17-20}$

The statistical analyses were performed using the Statistical Package for the Social Sciences (SPSS, IBM SPSS Statistics, Armonk, NY,US) software, version 25.0. The data are presented as means with standard deviations or frequencies with percentages. Data normality was assessed using the KolmogorovSmirnov test of normality. The Man-Whitney U test was used to compare latency and amplitude in the case and control groups. Analysis of Variance (ANOVA) was used to compare latency and amplitude in the sub-groups of the population with HL. The Student $t$ test was used to compare the ages of the groups. The Fisher exact test was used for the comparisons of the gender distribution, to evaluate the percentage of the presence of early biphasic P1-N1complex in the case and control groups, and the percentage of the same complex in the sub-groups of the population with HL. Given that the sample was numerically small,l $p$-values $\leq 0.01$ were considered statistically significant.

\section{Results}

The case group consisted of 20 subjects (65\% male and $35 \%$ female) with MPSHL and a mean age of 31.1 years (range: 18 to 79 years). 
Table 1 Patients in the case group

\begin{tabular}{|c|c|c|c|c|c|c|}
\hline CASES & EAR & P1 (ms) & $\mathrm{N} 1$ (ms) & P1-N1 ( $\mu \mathrm{v})$ & PTA dBHL & Etiology \\
\hline \multirow[t]{2}{*}{ Case-A } & Dx & 12.4 & 21.6 & 45.6 & 87.5 & \multirow[t]{2}{*}{ Vascular } \\
\hline & Sx & 16 & 22.8 & 71.6 & 88 & \\
\hline \multirow[t]{2}{*}{ Case-B } & Dx & \multirow{2}{*}{\multicolumn{3}{|c|}{ Absent bilateral cVemp responses }} & $>90$ & \multirow[t]{2}{*}{ Bacterial meningitis } \\
\hline & Sx & & & & $>90$ & \\
\hline \multirow[t]{2}{*}{ Case-C } & Dx & 13 & 21.4 & 44.5 & 88.6 & \multirow[t]{2}{*}{ Vascular } \\
\hline & $5 x$ & 13.5 & 23.9 & 39.2 & $>90$ & \\
\hline \multirow[t]{2}{*}{ Case-D } & Dx & 13.9 & 25.4 & 42.8 & 76 & \multirow[t]{2}{*}{ Vascular } \\
\hline & sx & 14.7 & 24.7 & 109 & 70 & \\
\hline \multirow[t]{2}{*}{ Case-E } & Dx & \multirow{2}{*}{\multicolumn{3}{|c|}{ Absent bilateral cVemp responses }} & 82.5 & \multirow[t]{2}{*}{ Viral } \\
\hline & Sx & & & & $>90$ & \\
\hline \multirow[t]{2}{*}{ Case-F } & Dx & 14.3 & 22.8 & 79.4 & $>90$ & \multirow[t]{2}{*}{ Viral } \\
\hline & Sx & 14.6 & 22.6 & 105.6 & $>90$ & \\
\hline \multirow[t]{2}{*}{ Case-G } & Dx & \multirow{2}{*}{\multicolumn{3}{|c|}{ Absent bilateral cVemp responses }} & $>90$ & \multirow[t]{2}{*}{ Viral } \\
\hline & Sx & & & & $>90$ & \\
\hline \multirow[t]{2}{*}{ Case- $\mathrm{H}$} & Dx & 19.3 & 28.3 & 34.4 & 75 & \multirow[t]{2}{*}{ Congenital } \\
\hline & $5 x$ & 10.2 & 23.2 & 28.6 & 72.5 & \\
\hline \multirow[t]{2}{*}{ Case-I } & Dx & 13.5 & 21.3 & 13.6 & 46 & \multirow[t]{2}{*}{ Vascular } \\
\hline & Sx & 14 & 21.3 & 18.7 & 55 & \\
\hline \multirow[t]{2}{*}{ Case-J } & Dx & 13.4 & 23 & 116.1 & $>90$ & \multirow[t]{2}{*}{ Viral } \\
\hline & Sx & 17.9 & 26.6 & 106.6 & $>90$ & \\
\hline \multirow[t]{2}{*}{ Case-K } & Dx & 19.8 & 26.5 & 69.7 & $>90$ & \multirow[t]{2}{*}{ Congenital } \\
\hline & Sx & 17.5 & 25.8 & 28.4 & $>90$ & \\
\hline Case-L & Dx & 13 & 19.8 & 72.4 & 67.5 & Vascular \\
\hline & Sx & Absent $\mathrm{cl}$ & esponse & & 88.4 & \\
\hline Case-M & Dx & Absent $\mathrm{cl}$ & esponses & & 90 & Vascular \\
\hline & Sx & 13.2 & 24.3 & 69.7 & 75.5 & \\
\hline Case-N & $\mathrm{Dx}$ & 12.4 & 21.3 & 16.6 & 64.8 & Congenital \\
\hline & Sx & 13 & 22 & 33.5 & 69 & \\
\hline Case-O & Dx & 13.2 & 24.3 & 116 & 78.7 & Vascular \\
\hline & $S x$ & Absent $\mathrm{cl}$ & esponse & & $>90$ & \\
\hline Case-P & Dx & 12.7 & 22.3 & 74.3 & 62.5 & Congenital \\
\hline & Sx & 13.2 & 24.1 & 69.5 & 63.7 & \\
\hline Case-Q & $\mathrm{Dx}$ & 18.4 & 24.5 & 62.4 & 87 & Congenital \\
\hline & Sx & 17 & 26.2 & 54.1 & 85 & \\
\hline Case-R & $\mathrm{Dx}$ & Absent bi & cVemp res & & $>90$ & Bacterial meningitis \\
\hline & Sx & & & & 90 & \\
\hline Case-S & Dx & 13.4 & 23.5 & 37.7 & 72.4 & Congenital \\
\hline & $5 x$ & 14 & 25 & 41.3 & 83.2 & \\
\hline Case-T & Dx & 16.5 & 21.4 & 73.3 & 87 & Vascular \\
\hline & $5 x$ & 12.8 & 22.5 & 69.6 & $>90$ & \\
\hline
\end{tabular}

Abbreviations: dBHL, decibels hearing level; Dx, Right; PTA, pure tone average; Sx, Left.

Notes: P1 (ms) and M1 (ms), latency of each of the biphasic complexes in milliseconds; P1-N1 ( $\mu \mathrm{v})$, amplitude of the biphasic complexes in microvolts; PTA dBHL, pure tone average of the hearing threshold at 500, 1,000, 2,000, and 4,000 Hz. 
Table 2 Cervical vestibular-evoked myogenic potentials in the case and control groups

\begin{tabular}{|l|l|l|l|}
\hline & Case group & Control group & $p$-value \\
\hline $\begin{array}{l}\text { Presence of } \\
\text { CVEMPs, n (\%) }\end{array}$ & $30 / 30(100 \%)$ & $29 / 40(72.5 \%)$ & 0.001 \\
\hline $\begin{array}{l}\text { P1 } \\
(\mathrm{ms} ; \text { mean } \pm \text { SD) }\end{array}$ & $14.74 \pm 1.71$ & $14.85 \pm 1.70$ & 0.858 \\
\hline $\begin{array}{l}\text { N1 } \\
(\mathrm{ms} ; \text { mean } \pm \text { SD) }\end{array}$ & $23.76 \pm 1.70$ & $29.63 \pm 2.19$ & 0.422 \\
\hline $\begin{array}{l}\text { P1-N1 } \\
(\mu \mathrm{v} ; \text { mean } \pm \text { SD) }\end{array}$ & $60.84 \pm 29.63$ & $77.53 \pm 29.63$ & 0.075 \\
\hline
\end{tabular}

Abbreviations: cVemps, cervical vestibular-evoked myogenic potentials; $\mathrm{SD}$, standard deviation.

Notes: P1 (ms) and M1 (ms), latency of each of the biphasic complexes in milliseconds; P1-N1 ( $\mu \mathrm{v})$ amplitude of the biphasic complexes in microvolts.

The control group consisted of 15 subjects (60\% male and $40 \%$ female), with normal hearing and an average age of 27.2 years (range: 15 to 44 years). There were no statistically significant differences regarding age and gender $(p=0.092$; $p=0.762$ respectively) between the case and control groups.

The patients of the study group had PTA thresholds between $44.5 \mathrm{~dB} \mathrm{HL}$ and $90 \mathrm{~dB} \mathrm{HL}$ or higher.

The subjects in the control group had normal hearing thresholds, lower or equal to $20 \mathrm{~dB} \mathrm{HL}$.

The study was performed on 35 subjects (70 ears), and it highlighted the presence of the early biphasic P1-N1 complex in 29 out of 40 (71.5\%) ears in the study group, while in the control group, the presence of the P1- N1 complex was detected all of the 30 ears examined. This data was statistically significant $(p=0.001)$.

There were no statistically significant differences in the comparison between the latencies and amplitude of the P1-N1 complex in the case and control groups(-Table 2 ).

The case group was divided into four subgroups based on the etiology. The study showed that the patients with a history of bacterial meningitis did not have the biphasic P1-N1complex. This complex appeared in all of the patients with congenital HL, while it was only present in some patients with HL caused by virus (50\%). The biphasic complex was monolaterally absent in some patients with high vascular and thrombophilic risk (-Table 1). Regarding the presence or absence of cVEMPs among the four subgroups of patients with $\mathrm{HL}$, the data were statistically significant $(p<0.001)$. The comparison between the latencies and amplitude of the P1-N1 complex in the sub-groups of patients with MPSHL was not statistically significant (-Table 3).

\section{Discussion}

Today, the number of publications concerning vestibularevoked myogenic potentials (VEMPs) is rapidly increasing. This fact suggests that many clinicians and researchers have recognized clinical and scientific values of VEMPs that have clinical application in Meniere disease, vestibular neuritis, benign paroxysmal positional vertigo, vestibular migraine and central vertigo/dizziness. ${ }^{17,21-23}$ The analysis of utricle and saccule activity through VEMPs is not influenced by hypoacusis. Vestibular-evoked myogenic potentials are activated by a sound stimulus transmitted either by bone or by air, which sets in motion the ossicular chain and the intralabirintic liquids. The endolymph will activate the vestibular receptors, if functioning properly. The only case in which the hypoacusis can alter the vestibular response is otosclerosis, even if the utricle and saccule are perfectly functional. In this case, the transmission of sound at the level of the ossicular chain is blocked and therefore the endolymph cannot be moved. ${ }^{23-25}$ Different studies showed vestibular dysfunction in patients with sensorineural $\mathrm{HL}$, especially children.,24 There are few relevant studies on the association between vestibular deficit and MPSHL in adults. Disorders of the inner ear may result in a variety of manifestations, including vertigo, spatial disorientation, blurred vision, impaired articulation, and hearing impairment. Considering the diversity of clinical symptoms associated with PSHL with otolithic dysfunction, it may be frequently misdiagnosed, and objective means of testing the function of otolithic organs should be recommended for hearing-impaired patients. ${ }^{2}$ There is no correlation between the latency and amplitude of the early biphasic P1-N1 complex and the patient's age. The dysfunction can be anywhere in the VEMP reflex path. In a recent investigation, Xu et $\mathrm{al}^{25}$ detected cVEMP responses in $44.4 \%$ of ears in patients with PSHL, while the response rate was of $100 \%$ in healthy subjects. These data demonstrate that patients with PSHL have a high incidence of damage to the otolithic organs. Similarly, Xu et $\mathrm{al}^{24}$ investigated VEMP results in 43 children with PSHL and 20 healthy children, and found that cVEMPs were induced in $61.9 \%$ of ears in children with PSHL, while the response rate in the healthy children was of $100 \%$. The literature data agree with the results of our study (cVEMP response in $71.5 \%$ of ears),

Table 3 Cervical vestibular-evoked myogenic potentials in the sub-groups of cases

\begin{tabular}{|l|l|l|l|l|l|}
\hline & Bacterial meningitis & Vascular & Congenital & Viral & $p$-value \\
\hline Presence of cVEMPs n (\%) & $0 / 4(0 \%)$ & $13 / 16(71.25 \%)$ & $12 / 12(100 \%)$ & $4 / 8(50 \%)$ & $<0.001$ \\
\hline P1 $(m s ;$ mean \pm SD) & $I$ & $13.82 \pm 1.22$ & $15.07 \pm 1.96$ & $15.05 \pm 3.15$ & 0.36 \\
\hline N1 $(m s ;$ mean \pm SD) & $/$ & $22.67 \pm 1.70$ & $24.39 \pm 2.07$ & $23.75 \pm 1.90$ & 0.09 \\
\hline P1-N1 $(\mu v ;$ mean \pm SD) & $/$ & $60.4 \pm 30.57$ & $45.87 \pm 19.32$ & $101.925 \pm 15.74$ & 0.02 \\
\hline
\end{tabular}

Abbreviations: cVemps, cervical vestibular-evoked myogenic potentials; SD, standard deviation.

Notes: P1 (ms) and M1 (ms), latency of each of the biphasic complexes in milliseconds; P1-N1 ( $\mu \mathrm{v})$, amplitude of the biphasic complexes in microvolts. 
considering also the fact that we have included subjects with moderate hearing loss. Disappearance and impairment of VEMPs could be observed after a cochlear implant (CI), and the waveforms of ocular VEMPs (oVEMPs) and cVEMPs could reflect the degree of damage to the utricle and saccule caused by the $\mathrm{CI}$ and other related factors. ${ }^{26,27}$

Another aim of our study is to study the correlation between the etiology of HL and a possible alteration in labyrinthine function. The limitation of the present study is that in these subgroups the number of patients is too small, and, in the future, we hope to enroll a higher number of patients. With the diagnosis of HL after a viral pathology in childhood, a PCR investigation could be performed for the detection of viral antigens. The most recent works in the literature on deafness that arose after exanthematous disease are all based on the diagnosis made only through the anamnestic investigation. ${ }^{13}$ For the deafness of possible vascular origin, we limited ourselves to a numerous series of risk factors that the patient had to possess to be included in the study population. For the division into subgroups we relied on previous studies in the literature. ${ }^{12-16}$

In the cases of HL due to a bacterial-viral cause, it is easier to find a concurrent vestibular disorder to a cochlear deficit, because of the close anatomical relationship that occurs between the two organs and the similarity of the receptor cells inside them. In the same way, in the case of meningitis, the close anatomical relationship with the cranial cavity plays a key role. ${ }^{12}$ In cases of postmeningitis bacterial deafness, cVEMPs were induced in $0 \%$ of ears, while the response rate was of $50 \%$ in the deafness of viral origin. In order to obtain a greater level of significance, these data require a greater sample. In the study by Tsubota et al, ${ }^{28}$ the virus was detected in the endolymph and in the perilymph. The proposed mechanisms of sensorineural HL include atrophy of hair cells in the organ of Corti and stria vascularis, and damage to the myelin sheath around the vestibulocochlear nerve. Vestibular dysfunction has also been reported in patients with mumps, suggesting that the eighth nerve is involved in the pathology because the utricle, saccule, and semicircular canals are not affected. ${ }^{13,28}$

The response to cVEMPs is present in $100 \%$ of patients with non-syndromic congenital HL. In these patients, we identified a genetic mutation of the gap junction $\beta 2$ (GJB2) gene, accounting for $50 \%$ of the cases of non-syndromic autosomal recessive HL. First described in 1997, GJB2 encodes connexin 26 , a gap junction protein critical to the potassium-ion channels of the cochlea. ${ }^{7,14,29}$ These data suggest that the mutation of the GJB2 gene does not damage the peripheral saccular receptor or the function of the inferior vestibular nerve. More studies on vestibular function in congenital deafness caused by intrauterine infections (cytomegalovirus, rubella and toxoplasmosis) would be useful.

The response to cVEMPs was monolaterally absent in 3 patients with high vascular and thrombophilic risk, coinciding with the ear with major hearing loss. This can direct us to identify a selective damage to the cochleo-vestibular artery, which vascularizes the cochlea, the macula of the saccule, and the ampulla of the posterior semicircular canal. Future studies using the oVEMPs or the video head impulse test (VHIT) are needed. Thanks to these non-invasive tests, we can identify ischemic damage to the anterior vestibular artery, which vascularizes the macula of the utricle and the ampulla of the superior and lateral semicircular canals, or damage to the labyrinthine artery, to the common branch of the vestibulocochlear artery, and to the anterior vestibular artery.

\section{Conclusion}

The use of cVEMPs is essential in the diagnosis of saccular dysfunction in patients with MPSHL. The labyrinthine function should receive attention in the diagnosis and treatment of MPSHL. This is useful to identify and intervene promptly in cases of vestibular disorders, which could endanger the process of integration of the critical sensory stimuli for a correct posture and locomotion. ${ }^{30-34}$ The present study aims to increase the current knowledge about the etiopathogenesis of sensorineural HL, which is often of unknown nature, ${ }^{5}$ to set the correct therapeutic management to improve the quality of life of the patient.

Informed consent

Informed consent was obtained from every participant included in the study.

Conflicts of interest

The authors have none to declare.

\section{Ethical approval}

All of the procedures performed in the present study were in accordance with the ethical standards of our institution and/or of the national research committee, as well as with the 1964 Helsinki declaration and its later amendments or comparable ethical standards.

\section{References}

1 Singh S, Gupta RK, Kumar P. Vestibular evoked myogenic potentials in children with sensorineural hearing loss. Int J Pediatr Otorhinolaryngol 2012;76(09):1308-1311. Doi: 10.1016/j. ijporl.2012.05.025

2 Zhou Y, Wu Y, Wang J. Otolithic organ function in patients with profound sensorineural hearing loss. JOtol 2016;11(02):73-77

3 Niu X, Zhang Y, Zhang Q et al. The relationship between hearing loss and vestibular dysfunction in patients with sudden sensorineural hearing loss. Acta Otolaryngol 2016;136(03):225-231. Doi: 10.3109/00016489.2015.1110750

4 Toumpas CJ, Clark J, Harris A, Beswick R, Nourse CB. Congenital cytomegalovirus infection is a significant cause of moderate to profound sensorineural hearing loss in Queensland children. JPaediatr Child Health 2015;51(05):541-544. Doi: 10.1111/jpc.12776

5 Gazia F, Abita P, Alberti G, et al. NICU Infants \& SNHL: Experience of a western Sicily tertiary care centre. Acta Med Mediter 2019;35 (02):1001-1007

6 Galletti B, Mannella VK, Santoro R, et al. Ear, nose and throat (ENT) involvement in zoonotic diseases: a systematic review. J Infect Dev Ctries 2014;8(01):17-23. Doi: 10.3855/jidc.4206

7 Amorini M, Romeo P, Bruno R, et al. Prevalence of DeafnessAssociated Connexin-26 (GJB2) and Connexin-30 (GJB6) Pathogenic Alleles in a Large Patient Cohort from Eastern Sicily. Ann Hum Genet 2015;79(05):341-349. Doi: 10.1111/ahg.12120 
8 Galletti B, Mannella VK, Santoro R, et al. Malignant external otitis. A case series from an Italian Tertiary-Care Hospital. Acta Med Mediter 2014;30(06):1317-1323

9 Freni F, Mannella VK, Cammaroto G, Azielli C, Cappuccio C, Galletti F. Classic and reversal steps stapedotomy performed with $\mathrm{CO} 2$ laser: a comparative analysis. Eur Arch Otorhinolaryngol 2014; 271(05):981-986. Doi: 10.1007/s00405-013-2500-6

10 Galletti F, Cammaroto G, Galletti B, Quartuccio N, Di Mauro F, Baldari S. Technetium-99m ( $\left.{ }^{99} \mathrm{mTc}\right)$-labelled sulesomab in the management of malignant external otitis: is there any role? Eur Arch Otorhinolaryngol 2015;272(06):1377-1382. Doi: 10.1007/ s00405-014-2938-1

11 Trovato MC, Ruggeri RM, Guzzo E, et al. Expression of P53 and isoforms in bening and malignant lesions of the head and neck. Histol Histopathol 2017;32(04):371-377. Doi: 10.14670/HH-11-802

12 Karanja BW, Oburra HO, Masinde P, Wamalwa D. Risk factors for hearing loss in children following bacterial meningitis in a tertiary referral hospital. Int J Otolaryngol 2013;2013:354725

13 Cohen BE, Durstenfeld A, Roehm PC. Viral causes of hearing loss: a review for hearing health professionals. Trends Hear 2014; 18:2331216514541361. Doi: 10.1177/2331216514541361

14 Funamura JL. Evaluation and management of nonsyndromic congenital hearing loss. Curr Opin Otolaryngol Head Neck Surg 2017;25(05):385-389. Doi: 10.1097/MOO.0000000000000398

15 Marcucci R, Alessandrello Liotta A, Cellai AP, et al. Cardiovascular and thrombophilic risk factors for idiopathic sudden sensorineural hearing loss. J Thromb Haemost 2005;3(05):929-934

16 Passamonti SM, Di Berardino F, Bucciarelli P, et al. Risk factors for idiopathic sudden sensorineural hearing loss and their association with clinical outcome. Thromb Res 2015;135(03):508-512. Doi: $10.1016 / j$.thromres.2015.01.001

17 Murofushi T. Clinical application of vestibular evoked myogenic potential (VEMP). Auris Nasus Larynx 2016;43(04):367-376

18 Papathanasiou ES, Murofushi T, Akin FW, Colebatch JG. International guidelines for the clinical application of cervical vestibular evoked myogenic potentials: an expert consensus report. Clin Neurophysiol 2014;125(04):658-666

19 Colebatch JG, Halmagyi GM, Skuse NF. Myogenic potentials generated by a click-evoked vestibulocollic reflex. J Neurol Neurosurg Psychiatry 1994;57(02):190-197

20 Colebatch JG, Halmagyi GM. Vestibular evoked potentials in human neck muscles before and after unilateral vestibular deafferentation. Neurology 1992;42(08):1635-1636

21 Galletti B, Gazia F, Freni F, Nicita RA, Bruno R, Galletti F. Chronic Otitis Media Associated with Cholesteatoma in a Case of the SayBarber-Biesecker-Young-Simpson Variant of Ohdo Syndrome. Am J Case Rep 2019;20:175-178
22 Ciodaro F, Mannella VK, Nicita RA, et al. Therapeutic efficacy of the Galletti-Contrino manoeuvre for benign paroxysmal positional vertigo of vertical semicircular canals in overweight subjects. Eur Arch Otorhinolaryngol 2018;275(10):2449-2455. Doi: 10.1007/ s00405-018-5086-1

23 Catalano N, Cammaroto G, Galletti B, et al. The role of cVEMPs and vHIT in the evaluation of otosclerosis and its eventual vestibular impairment: preliminary findings. B-ENT 2017;13 (01, Suppl 27):31-36

$24 \mathrm{Xu}$ XD, Zhang Q Hu J, et al. The hidden loss of otolithic function in children with profound sensorineural hearing loss. Int J Pediatr Otorhinolaryngol 2015;79(06):852-857. Doi: 10.1016/j. ijporl.2015.03.017

$25 \mathrm{Xu}$ XD, Ding CR, Yu J, et al. The hidden dysfunction of otolithic organs in patients with profound sensorineural hearing loss. Hear Res 2016;331:41-46. Doi: 10.1016/j.heares.2015.10.006

$26 \mathrm{Xu} \mathrm{XD}$, Zhang XT, Zhang Q Hu J, Chen YF, Xu M. Ocular and cervical vestibular-evoked myogenic potentials in children with cochlear implant. Clin Neurophysiol 2015;126(08):1624-1631. Doi: 10.1016/j.clinph.2014.10.216

27 Mento C, Galletti F, Freni F, et al. The role of temperament in traumatic hearing loss: a single case study of a cochlearimplanted patient. Int J Adolesc Med Health 2016;28(01): 107-113. Doi: 10.1515/ijamh-2014-0075

28 Tsubota M, Shojaku H, Ishimaru H, Fujisaka M, Watanabe Y. Mumps virus may damage the vestibular nerve as well as the inner ear. Acta Otolaryngol 2008;128(06):644-647. Doi: 10.1080/ 00016480701646305

29 Kelsell DP, Dunlop J, Stevens HP, et al. Connexin 26 mutations in hereditary non-syndromic sensorineural deafness. Nature 1997; 387(6628):80-83

30 Ciodaro F, Freni F, Mannella VK, et al. Use of 3D Volume Rendering Based on High-Resolution Computed Tomography Temporal Bone in Patients with Cochlear Implants. Am J Case Rep 2019; 20:184-188

31 Leo A, Naro A, Cannavò A, et al. Could autonomic system assessment be helpful in disorders of consciousness diagnosis? A neurophysiological study. Exp Brain Res 2016;234(08):2189-2199. Doi: 10.1007/s00221-016-4622-8

32 Rizzo G, Milardi D, Bertino S, et al. The Limbic and Sensorimotor Pathways of the Human Amygdala: A Structural Connectivity Study. Neuroscience 2018;385:166-180

33 Chillemi G, Calamuneri A, Quartarone A, et al. Endogenous orientation of visual attention in auditory space. JAdv Res 2019;18:95-100

34 Portaro S, Naro A, Cacciola A, et al. Adult-Onset Walking-Upstairs Dystonia. JClin Neurol 2019;15(01):122-124 